\title{
Energy-Efficient CMOS Large-Load Driver Circuit with the Complementary Adiabatic/Bootstrap (CAB) Technique for Low-Power TFT-LCD System Applications
}

\author{
G. Y. Liu, N. C. Wang and J. B. Kuo* \\ Dept of Electrical Engineering, BL-528 \\ National Taiwan University \\ Taipei, Taiwan 106-17 \\ Email: j.kuo@ieee.org
}

*J. B. Kuo is on leave from NTUEE, with SFU in Canada

\begin{abstract}
This paper reports an energyefficient CMOS large-load driver circuit with the complementary adiabatic/bootstrap (CAB) technique for low-power TFT-LCD system applications. Using two differential inputs and via dual paths for bootstrap and energy recovery to $V_{D D}$ /ground, this $C A B$ load driver with an output load of $30 \mathrm{pF}$ and operating at $5 \mathrm{~V}$, provides a high-speed performance, consuming $60 \%$ less power as compared to the adiabatic/bootstrapped driver with single-path for bootstrap and energy recovery.
\end{abstract}

\section{INTRODUCTION}

Large-load driver circuit is an important component in a modern TFT-LCD system, where high speed and low power are the goals [1]. Bootstrap technique has been used in the low-voltage driver circuits to enhance speed performance of a VLSI system [2]-[4]. Energy efficiency of a large-load driver circuit is important in determining the power consumption of a TFT-LCD system. Adiabatic technique has been used to raise the energy efficiency of low-voltage VLSI circuits [5]. Recently, the adiabatic technique combining with the bootstrap technique to generate an energy efficient large-load driver circuit for lowvoltage VLSI systems has been reported [6]. In this paper, an energy-efficient CMOS largeload driver circuit with the complementary adiabatic/bootstrap (CAB) technique via dual paths for bootstrap and energy recovery to $\mathrm{V}_{\mathrm{DD}}$ /ground, for low-power TFT-LCD system applications is described. In the following sections, this CMOS CAB load driver is described first, followed by performance and discussion.

\section{CAB Load Driver}

Fig. 1 shows the CMOS load driver using the complementary adiabatic/bootstrap (CAB)

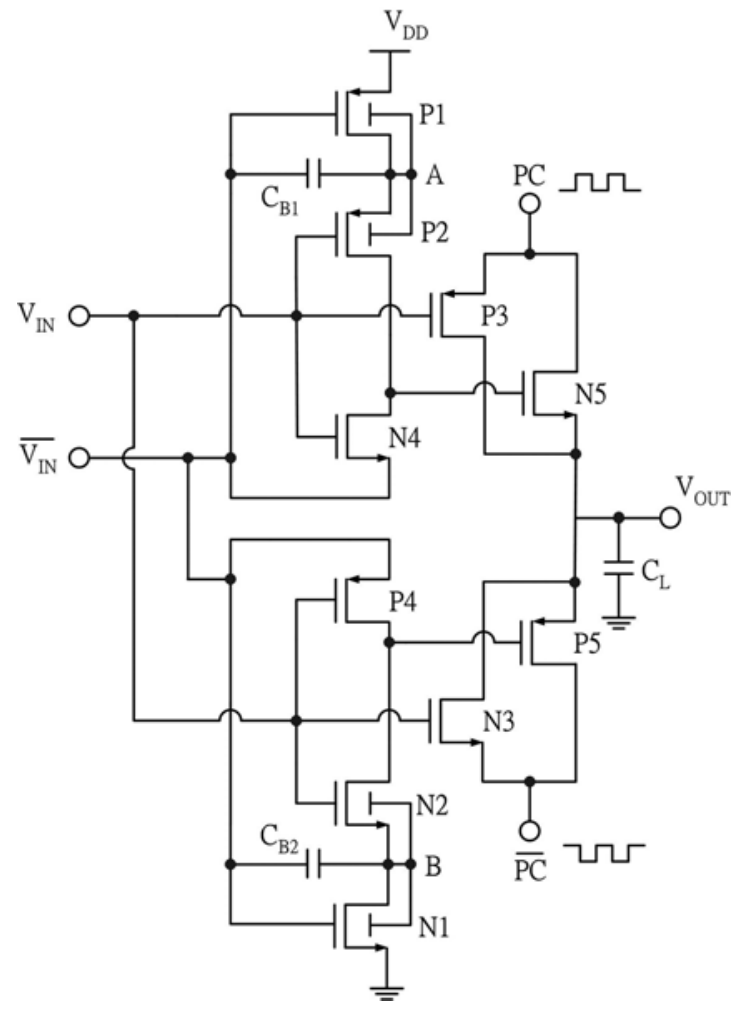

Fig. 1. The CMOS CAB large-load driver circuit using the dual-path bootstrap/energy recovery technique.

technique via dual paths for bootstrap and energy recover to $V_{D D}$ /ground. As shown in the figure, $\mathrm{P} 1, \mathrm{P} 2, \mathrm{P} 3, \mathrm{~N} 4, \mathrm{~N} 5$, and the capacitor $\mathrm{C}_{\mathrm{B} 1}$ form the pull-up segment and N1, N2, N3, $\mathrm{P} 4, \mathrm{P} 5$, and the capacitor $\mathrm{C}_{\mathrm{B} 2}$ form the pulldown segment. In the pull-up segment, $\mathrm{P} 1, \mathrm{P} 2$, $\mathrm{N} 4, \mathrm{~N} 5$ and $\mathrm{C}_{\mathrm{B} 1}$ are used to implement the bootstrap technique for enhancing the driving capability of N5 during the pull-up transient. By the same token, in the pull-down segment, N1, $\mathrm{N} 2, \mathrm{P} 4, \quad \mathrm{P} 5$ and the capacitor $\mathrm{C}_{\mathrm{B} 2}$ are implemented to increase the driving capability of P5 using the bootstrap technique during the pull-down transient. Two differential inputs have been adopted in the circuits. Instead of 

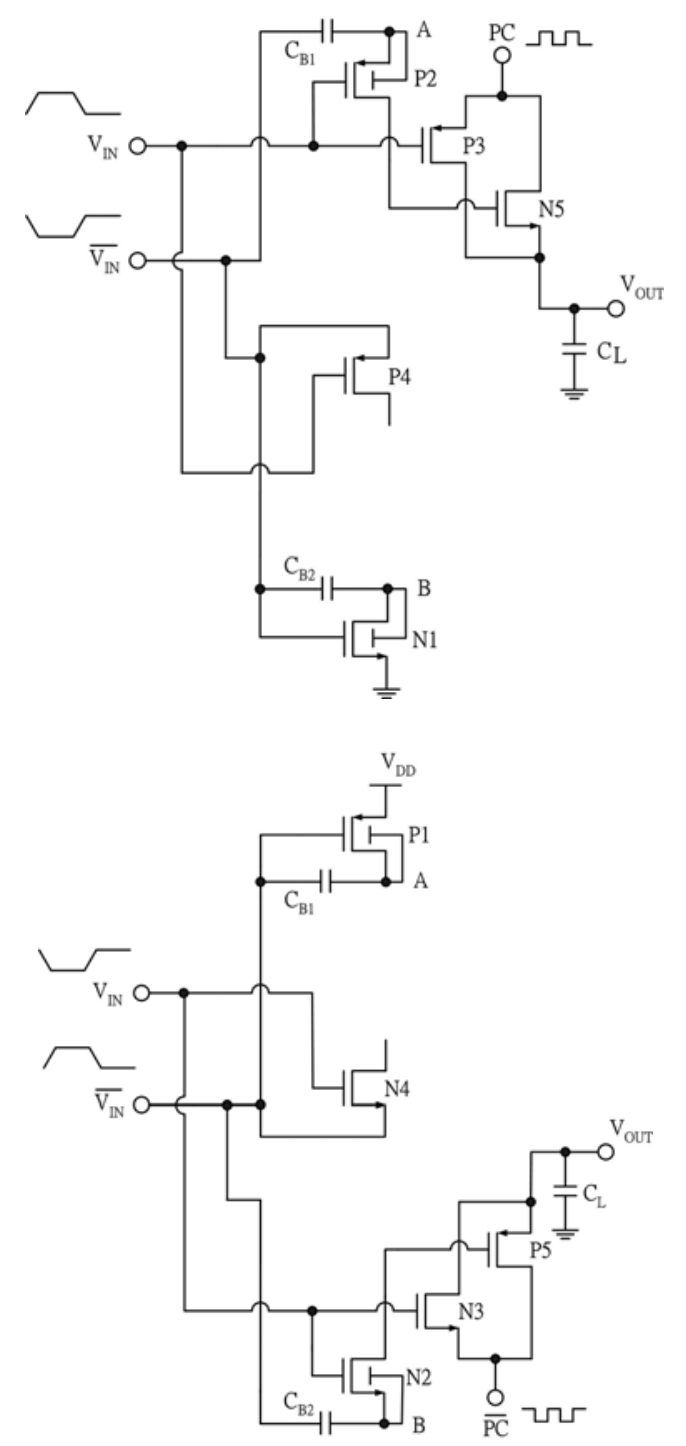

Fig. 2. Equivalent circuits of the $C M O S C A B$ load driver using the dual-path bootstrap/energy recovery technique when $V_{\text {IN }}$ is low (a) high (b).

the single path for bootstrap/energy recovery to $V_{D D}$ as in the previous approach [6], dual paths for energy recovery to $V_{D D}$ and ground have been adopted to increase the energy efficiency of this circuit.

The operation of this CMOS CAB load driver circuit is divided into two modes- when $V_{\text {IN }}$ is low (1) and high (2). When $V_{\mathbb{I N}}$ is low (1), the pull-down segment is inactivated except that the bootstrap capacitor $\mathrm{C}_{\mathrm{B} 2}$ is charged with its left side to high and right side to OV, since $\mathrm{N} 1$ is on. At the same time, in the pull-up segment the bootstrap capacitor $\mathrm{C}_{\mathrm{B} 1}$ is floating with its right side disconnected from $V_{D D}$. In addition, the rest of the pull-up segment is

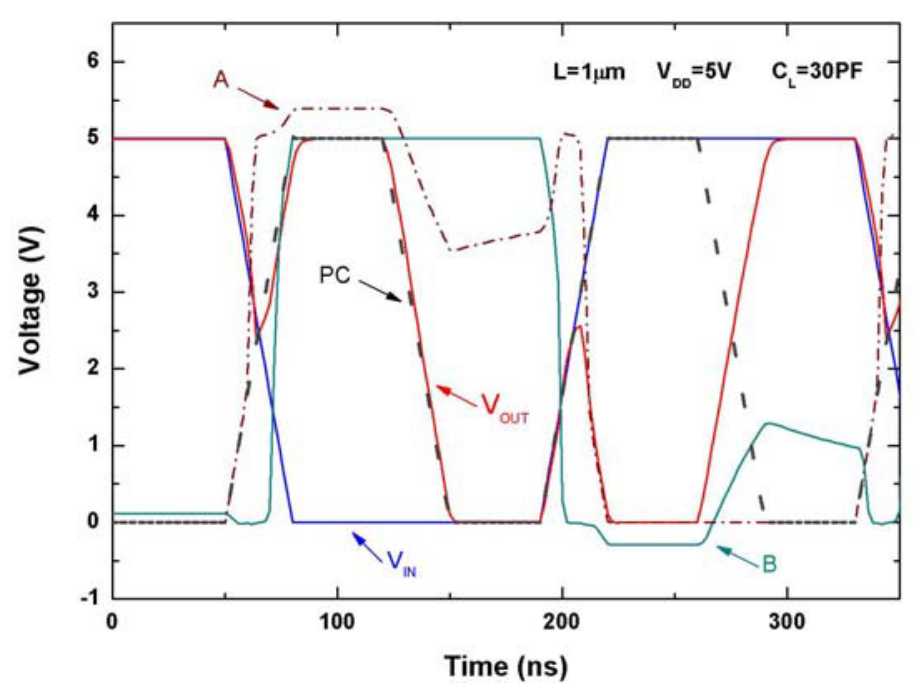

Fig. 3. Transient waveforms of the $5 \mathrm{~V}$ CMOS CAB load driver using the dual-path bootstrap/energy recovery technique.

ready for (1) energy recovery and (2) output operations. When PC switches from high to low, it is the energy recovery operation for the pull-up segment. Energy recovery is done from the output load $C_{L}$ via N5. When $P C$ is switching from low to high, it is the output operation period. During this period, the internal node $A$ may exceed $V_{D D}$ as shown in Fig. 3 owing to the bootstrap capacitor $\mathrm{C}_{\mathrm{B} 1}$. Therefore a large driving current of N5 ensures a high pull-up switching speed at the final output.

The operations of energy recovery and output for the case when $V_{\mathbb{I N}}$ is high are opposite to those described above for the case when $V_{\text {IN }}$ is low. When $V_{\text {IN }}$ is high, the pull-up segment is inactivated except the charge of the bootstrap capacitor $\mathrm{C}_{\mathrm{B} 1}$. In addition, energy recovery and output operations are done for the pull-down segment depending on PC* When $\mathrm{PC}^{*}$ turns from low to high, energy recovery is done from the output node via P5. When $\mathrm{PC}^{*}$ turns low, it is the output period for the pull-down segment, where internal B may go under $0 \mathrm{~V}$ due to the bootstrap capacitor $\mathrm{C}_{\mathrm{B} 2}$, which enhances the driving capability of P5 such that the pull-down speed of the output node could be enhanced. Owing to the implementation of the $C A B$ technique via the dual paths of bootstrap/energy recovery to $V_{D D}$ and ground, the energy efficiency and the 
speed of this large-load driver have been enhanced.

\section{Performance}

In order to assess the performance of this CMOS CAB large-load driver circuit with the dual-path energy recovery/bootstrap technique, a test circuit using a $5 \mathrm{~V}$ power supply voltage with a capacitive load of $30 \mathrm{pF}$ at the output node, based on a $0.35 \mu \mathrm{m}$ CMOS technology has been designed. The channel length of all devices in the circuit is $1 \mu \mathrm{m}$. Bootstrap capacitor $\mathrm{C}_{\mathrm{B} 1} / \mathrm{C}_{\mathrm{B} 2}$ of $0.5 \mathrm{pF} / 0.8 \mathrm{pF}$ has been used. Fig. 3 shows the transient waveforms of this load driver circuit at $5 \mathrm{~V}$. As shown in the figure, owing to the function of the bootstrap capacitors $C_{B 1} / C_{B 2}$, the internal node $A / B$ may

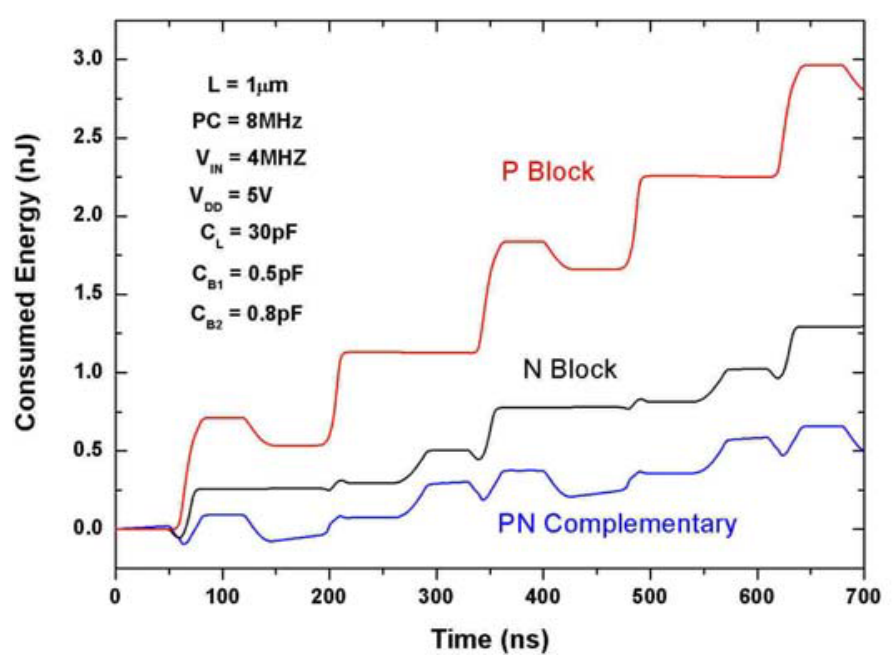

Fig. 4. Consumed energy of the 5V CMOS CAB large-load driver using the dual-path adiabatic/ bootstrap technique.

be higher/lower than $5 \mathrm{~V} / 0 \mathrm{~V}$. Thus the final output can be pulled to high/low at a high speed owing to the enhanced driving capability of N5/P5.

Fig. 4 shows the consumed energy of this $5 \mathrm{~V}$ CMOS CAB large-load driver using the dual-path adiabatic/bootstrap technique operating at $\mathrm{PC}$ of $8 \mathrm{MHz}$. Also shown in the figure are the results for the load drivers using the single-path adiabatic/bootstrap approach [6]. Note that $P$ block means the driver with the pull-up segment only and $\mathrm{N}$ block implies the one with the pull-down segment only. As shown in the figure, the CMOS CAB load driver with the dual-path bootstrap/energy recovery technique indicates a $60.3 \% / 82 \%$ reduction in the power consumption as compared to the one with $\mathrm{N}$ block/P block using the single-path bootstrap/energy recovery technique [6]. From this figure, the CMOS CAB load driver with the dual-path bootstrap/energy recovery technique is effective in raising the energy efficiency of the circuit.

\section{IV.DISCUSSION}

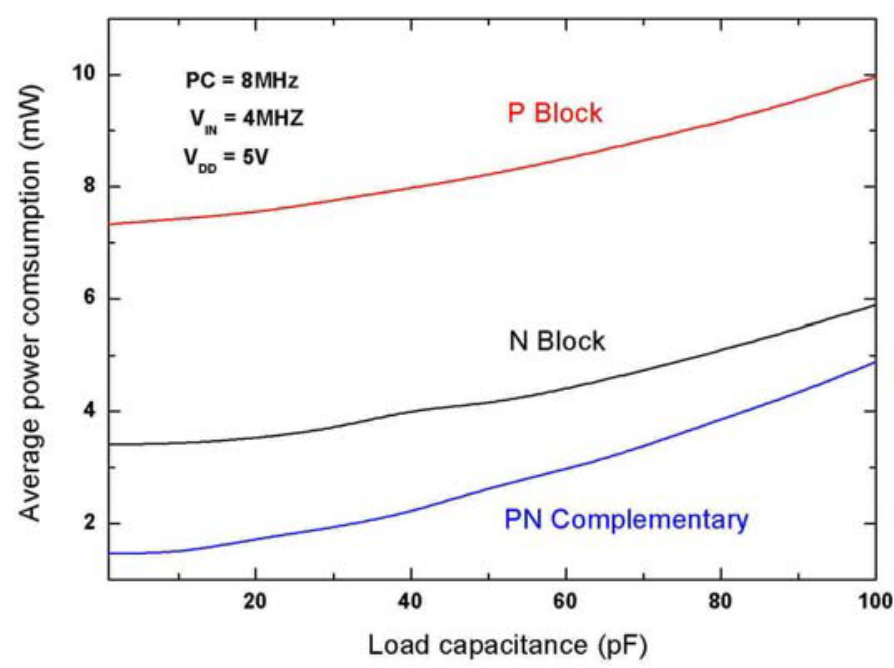

Fig. 5. Average power consumption versus load capacitance of the $5 \mathrm{~V}$ CMOS CAB load driver using the dual-path bootstrap/ energy recovery techniques

This CMOS CAB load driver using the dualpath bootstrap/energy recovery technique is especially advantageous when the load is large. Fig. 5 shows the average power consumption versus load capacitance of the CMOS CAB load driver using the dual-path bootstrap/energy recovery technique at the clock frequency of $8 \mathrm{MHz}$. Also shown in the figure are the results for the load driver using the single-path adiabatic/bootstrap approach [6]. As shown in the figure, at a load capacitance of $100 \mathrm{pF}$, the CMOS CAB load driver using the dual-path bootstrap/energy 


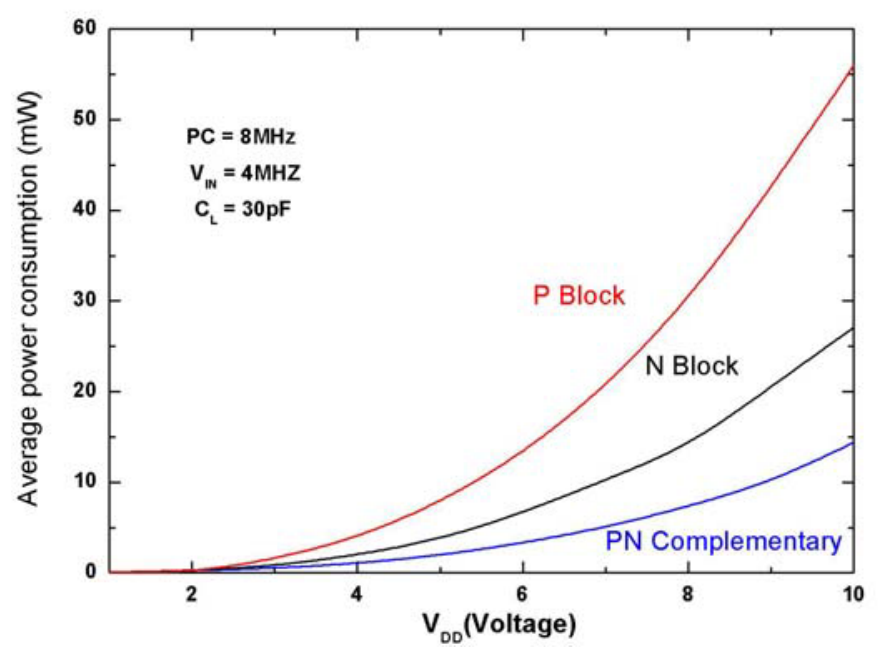

Fig. 6. Average power consumption versus power supply voltage of the CMOS $\mathrm{CAB}$ load driver using the dual-path bootstrap/energy recovery technique.

recovery technique offers a $16 \% / 50 \%$ reduction in the average power consumption as compared to the $\mathrm{N}$ block/P block using the single-path bootstrap/energy recovery technique [6].

Fig. 6 shows the average power consumption versus power supply voltage of the CMOS CAB load driver using the dual-path bootstrap/energy recovery technique with a load capacitance of $30 \mathrm{pF}$ at the clock frequency of $8 \mathrm{MHz}$. As shown in the figure, at the power supply voltage of $10 \mathrm{~V}$, the CMOS load driver with the dual-path bootstrap/ energy recovery technique offers a reduction of $42 \% / 73 \%$ in the average power consumption as compared to the $\mathrm{N}$ block/P block one using the single-path adiabatic/ bootstrap technique. Consequently, the CMOS $\mathrm{CAB}$ load driver using the adiabatic/bootstrap techniques is effective in high speed and energy efficiency.

\section{CONCLUSION}

In this paper, an energy-efficient CMOS large load driver circuit with the complementary adiabatic/bootstrap (CAB) technique for low-power TFT-LCD system applications has been reported. Using two differential inputs and via dual paths for bootstrap and energy recovery to $\mathrm{V}_{\mathrm{DD}}$ /ground, this $C A B$ load driver with an output load of
$30 \mathrm{pF}$ and operating at $5 \mathrm{~V}$, provides a highspeed performance, consuming $60 \%$ less power as compared to the adiabatic/ bootstrapped driver with single-path for bootstrap and energy recovery.

\section{ACKNOWLEDGMENT}

This project is supported under R.O. C. National Science Council Grant No. 83-2215E002-020.

\section{REFERENCES}

[1] T. Itakura, H. Minamizaki, T. Saito, T. Kuroda, "A 402-output TFT-LCD Driver IC with Power Control Based on the Number of Color Selected," IEEE J. Solid-State Circuits, Vol. 38, No. 3, pp. 503-510, March 2003.

[2] C. F. Law, K. S. Yeo, and R. S. Samir, "Sub-1V Bootstrapped CMOS Driver for Giga-Scale-Integration Era," Elec. Lett., Vol. 35, No. 5, pp. 392-393, 1999.

[3] K. S. Yeo, J. G. Ma, M. A. Do, "Ultra-LowVoltage Bootstrapped CMOS Driver for High Performance Applications," Elec. Lett., Vol. 36, No. 8, pp. 706-707, 2000.

[4] J. H. Lou and J. B. Kuo, "A 1.5V Full-Swing Bootstrapped CMOS Large CapacitiveLoad Driver Circuit Suitable for LowVoltage CMOS VLSI," IEEE J. Solid-State Circuits, Vol. 32, No. 1, pp. 119-121, 1997.

[5] Y. Zhang, H. H. Chen, and J. B. Kuo, "0.8V CMOS Adiabatic Differential Switch Logic Circuit Using Bootstrap Techniques for Low-Voltage Low-Power VLSI," Elec. Lett., Vol. 38, No. 24, pp. 1497-1499, 2002.

[6] H. P. Chen and J. B. Kuo, "A Low-Voltage CMOS Load Driver with the Adiabatic and Bootstrap Techniques for Low-Power System Applications," MWSCAS Proc., Hiroshima, July 2004. 